\title{
Usages des TIC et appropriations des médias en $p$ ériode de crise sociale
}

ICT uses and media appropriations in social crisis

Usos de las tecnologías de información y de comunicación (TIC) y apropiaciones

de los medios de comunicación en crisis social

Farrah Bérubé et Olivier Pulvar

\section{(2) OpenEdition}

Journals

\section{Édition électronique}

URL : http://journals.openedition.org/ctd/1904

DOI : $10.4000 /$ ctd. 1904

ISSN : 2491-1437

\section{Éditeur}

Chaire Unesco Pratiques émergentes en technologies et communication pour le développement

Édition imprimée

Date de publication : 12 novembre 2015

\section{Référence électronique}

Farrah Bérubé et Olivier Pulvar, « Usages des TIC et appropriations des médias en période de crise sociale », Communication, technologies et développement [En ligne], 2 | 2015, mis en ligne le 11 juin 2019, consulté le 22 juillet 2020. URL : http://journals.openedition.org/ctd/1904 ; DOl : https://doi.org/ $10.4000 /$ ctd. 1904

Ce document a été généré automatiquement le 22 juillet 2020

Communication, technologies et développement 


\title{
Usages des TIC et appropriations des médias en période de crise sociale
}

\author{
ICT uses and media appropriations in social crisis \\ Usos de las tecnologías de información y de comunicación (TIC) y apropiaciones \\ de los medios de comunicación en crisis social
}

Farrah Bérubé et Olivier Pulvar

1 L'ouvrage de référence Biografía del Caribe voit dans le passé de l'Amérique les fragments du mot démocratie. Il présente la mer des Caraïbes, qui a vu passer bien des ouragans violents, comme le théatre de luttes pour la démocratie du XVI ${ }^{\mathrm{e}}$ au $\mathrm{XX}^{\mathrm{e}}$ siècle (Arciniegas, 1984, p. 405). C'est dans l'histoire de ces luttes sociales, dans l'instauration des processus démocratiques dans divers pays de la grande région, que le courant latino-américain des cultural studies puise ses configurations premières. C'est également dans un dialogue à la fois constant et contradictoire avec les écoles de pensées occidentales (structuralistes français ou post-structuralistes, école de Frankfurt sur la théorie de la réception, avec les sémiotiques et les féministes, courants britannique et américain des cultural studies), que le courant latino-américain des cultural studies éclaire les productions symboliques et les expériences vécues à partir des réalités sociales latino-américaines (Trigo, 2004, p. 3).

2 Comment envisager par exemple, l'appropriation et les usages des médias sous des angles inhabituels comme ceux de la culture, de la politique, des médiations et de l'éducation des auditoires? Martín-Barbero (2012), souligne que le rôle central des médias dans ces sociétés ne peut être remis en question et qu'il semble disproportionné et paradoxal dans les pays de l'Amérique latine où les besoins de base en éducation et en santé n'ont pas encore été rencontrés. Il ajoute que les inégalités croissantes dans ces pays divisent la société et affaiblissent les raisons d'être et les mécanismes de communication.

3 À travers l'étude de mobilisations collectives s'étant jouées dans la zone des Caraïbes (les élections présidentielles mexicaines de 2012, la grève générale aux Antilles françaises en 2009), cet article se penche sur les usages et les appropriations des médias 
traditionnels et des technologies de l'information et de la communication (TIC) par des groupes minoritaires. Avant de discuter ces processus sur fond de crise sociale, il faut nécessairement présenter le rôle des médias dans le contexte des perturbations sociales observées.

\section{Les élections présidentielles mexicaines de 2012 à l'épreuve du journalisme citoyen}

Le Mexique est une République fédérale composée de 31 États et d'un District fédéral. Tous les six ans, les Mexicains élisent au suffrage universel direct à un tour et à la majorité le président de la République. Au début de l'été 2012, on annonce une campagne tranquille qui devrait signer le retour du Parti Révolutionnaire Institutionnel ou PRI. Le PRI a gouverné le Mexique sans interruption pendant 71 ans, de 1929 à 2000. Traînant derrière lui un passé d'autoritarisme et de corruption, le PRI a perdu le pouvoir le 2 juillet 2000 au profit du Parti d'Action Nationale ou PAN. Pour ces élections de 2012, le candidat à la présidence de la République du PRI, Enrique Peña Nieto, a en début de campagne, il est vrai, la faveur populaire, d'autant qu'il a été gouverneur de l'État de México durant les 6 années précédentes ${ }^{1}$.

\section{Des libertés d'expression et d'information}

\section{YouTube, l'effet papillon}

5 La tranquille campagne est perturbée le 11 mai 2012 alors que Nieto participe à une conférence à la Universidad Iberoamericana dans la capitale mexicaine. A cette occasion, des étudiants le questionnent sur les arrestations et les violations des droits humains dans la ville d'Atenco en 2006. La révolte d'Atenco désigne des manifestations contre lesquelles Nieto, alors gouverneur de l'État de México, avait ordonné le déploiement de 3000 policiers fédéraux. Cette action avait donné lieu à l'arrestation violente de 200 personnes, à plusieurs agressions sexuelles contre des femmes et au décès de deux jeunes personnes. À la réponse donnée par le candidat du PRI à la présidence de la République, les étudiants présents le huent et scandent les mots asesino $^{2}$ et fuera ${ }^{3}$. L'entourage de Nieto lui fait quitter le campus universitaire sur-lechamp. La sortie précipitée de Nieto sous une pluie d'insultes, est filmée et aussitôt diffusée sur YouTube ${ }^{4}$. Relevant du journalisme citoyen, ces images prises par des personnes présentes sur le campus à l'aide de leur cellulaire permettent une distanciation par rapport à l'information officielle; elles suscitent l'intérêt sur ce qui est important pour les émetteurs. Le jour même, les télévisions nationales du pays reprennent les images et les diffusent dans divers programmes et bulletins de nouvelles.

6 En réaction à l'événement transporté dans l'espace médiatique national, les dirigeants du PRI, ainsi que d'autres dirigeants de partis politiques alliés au PRI, arguent que la salle de conférence de la Universidad Iberoamericana était bondée d'un groupe minoritaire proche du parti de la gauche et non pas d'étudiants universitaires et que ceux-ci avaient cherché à créer un scandale. Les principales chaînes de télévision du pays reprennent cette idée qu'il ne s'agissait pas d'une expression authentique d'étudiants. La réplique des étudiants ne se fait pas attendre et prend la forme d'une 
nouvelle vidéo diffusée sur YouTube le 14 mai $2012^{5}$. Cette vidéo met en scène 131 étudiants tous présents lors de la conférence publique sur le campus de la Universidad Iberoamericana et qui montrent à la caméra leur carte étudiante prouvant leur statut. Les étudiants déclarent qu'ils n'ont pas été entraînés par quelconque groupe minoritaire proche du parti de la gauche ou tout autre parti et que leur geste n'était pas planifié.

7 La réponse des 131 étudiants de la Iberoamericana suscite un vent de sympathie partout dans le pays et rassemble autour d'un slogan \#YoSoy132, des milliers de Mexicains dénonçant en plein cœur de la campagne électorale, une situation nationale désastreuse et de violence où l'information est largement manipulée par les médias. Yo Soy 132 signifie " Je suis le $132^{e}$ ». Le mouvement vient de naître.

\section{Le manifeste de \#YoSoy132 pour la démocratisation des médias}

8 À la fin du mois de mai 2012, \#YoSoy132 s'organise et diffuse son manifeste également sous la forme d'une vidéo sur YouTube 6 . C'est par la diffusion d'abord du manifeste que le mouvement se fait connaître et qu'il peut rassembler, autour de ses idées, de plus en plus de Mexicains et pas que des étudiants. Les idées maitresses véhiculées dans le manifeste sont celles d'un mouvement non partisan, de la liberté d'expression des Mexicains et de la démocratisation des médias du pays afin que soit respecté le droit de tous les citoyens mexicains à être informés. Dans sa version originale du 23 mai, le document stipule au sujet de la démocratisation des médias :

[...] En esencia, nuestro movimiento busca la democratización de los medios de comunicación, con el fin de garantizar información transparente, plural e imparcial para fomentar una consciencia y pensamiento críticos. Es por eso que, exigimos competencia real en el mercado de medios de comunicación, en particular a lo referente al duopolio televisivo. Exigimos hacer del acceso a Internet un derecho constitucional efectivo en los términos que establece el Art. 1 de nuestra Carta Magna. Exigimos la instalación en todos los medios informativos radio, televisión y medios impresos, civiles que defiendan el interés público, como lo son la publicación de un Código de Ética del manejo informativo y la instauración de un ombudsman. Exigimos someter a concurso producciones para los canales públicos permisionarios a las distintas escuelas de Comunicación. Exigimos abrir el debate entre los jóvenes y los medios de comunicación sobre las demandas aquí expuestas $[\ldots]^{7}$.

9 Sur le point du droit des citoyens mexicains être informés, les sympathisants de \#YoSoy132 jugent que les liens entre la presse nationale et le pouvoir politique sont antidémocratiques au Mexique et que l'ensemble des médias du pays sont concentrés entre quelques mains. Les revendications majeures du mouvement concernent donc l'espace médiatique mexicain. Deux grands groupes médiatiques sont notamment visés, Televisa et TV Azteca, pour leur couverture partisane lors de la campagne présidentielle mexicaine. Dans ce contexte, \#YoSoy132 investit les nouvelles plateformes médiatiques afin de faire circuler ses idées.

\section{Une médiatisation d'opinion au coeur de la grève générale de 2009 aux Antilles françaises}

10 Le 20 janvier 2009, marque dans la conscience collective le moment de la cérémonie d'investiture du premier président noir des États-Unis, Barack Obama, pour son 
premier mandat. C'est la date également choisie par un Collectif contre l'exploitation outrancière (Liyannaj Kont Pwofitasyon_LKP), sur l'île française de la Guadeloupe, pour lancer un appel à la grève générale et déposer auprès des autorités publiques une plateforme de 146 revendications.

Réunis autour de 49 syndicats, partis politiques, associations socioculturelles et organisations diverses, des milliers de manifestants réclament une baisse du prix des carburants, des produits de première nécessité, des impôts et des taxes, ainsi qu'une hausse du salaire minimum de 200 euros. Des blocages affectent les activités dans plusieurs secteurs: l'éducation, les transports, le bâtiment, la santé, le tourisme, l'hôtellerie, ou encore ceux de la distribution de l'électricité et de l'eau. Les prémisses de cette agitation étaient déjà visibles un mois plus tôt (Calimia-Dinane, 2009), signe d'une mobilisation collective inédite qui allait paralyser durant huit semaines tous les secteurs socioéconomiques.

\section{Entre happy slapping et information continue du public}

\section{Canal 10, la proximité absolue?}

12 Rapidement, des négociations s'ouvrent, en présence de médias. La chaîne de télévision locale privée Canal 10 retransmet en direct et dans leur intégralité les discussions entre les représentants des pouvoirs publics, les parlementaires, les responsables des exécutifs régionaux, les socioprofessionnels et le Collectif. Le service public local d'audiovisuel qui lui, a cessé le travail, ne reprendra ses programmes normalement qu'après 6 semaines d'interruption.

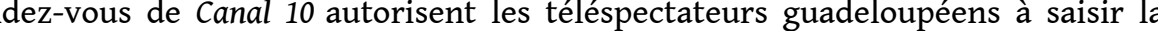
place, le rôle, les postures de chacun, de même que la nature et la forme de leurs rapports. L'expérience montre comment les parties en présence parviennent à trouver conjointement une méthode de travail, comment les discussions (en français et en créole) sont âpres, mais surtout, comment le préfet et ses collaborateurs quittent brutalement la table des négociations. Si l'incident signe la fin de l'expérience de télédiffusion des négociations, il marque aussi que le LKP et son leader, Elie Domota, ont choisi de faire des médias une ressource stratégique de leur action ${ }^{8}$.

De son côté, le Secrétaire d'État à l'Outre-mer, Yves Jégo, s'exprime sur les ondes de la première radio locale depuis Paris; avant de se rendre sur place. Sa venue favorise la médiatisation nationale du conflit, et malgré de nombreuses initiatives pour résoudre le conflit, la tension reste palpable. En réaction à une rumeur selon laquelle les chefs d'entreprises seraient prêts à assurer leur propre sécurité et à faire cesser les pressions exercées sur les entreprises, Élie Domota lance un avertissement sur Canal 10: «Si quelqu'un blesse un membre du LKP ou un manifestant guadeloupéen, il y aura des morts ", " Les békés prennent leurs dispositions, nous prenons les nôtres", "Si c'est la guerre civile que vous voulez et qu'il y a besoin de faire un Mai 67 [référence aux émeutes sanglantes liées aux évé nements du Groupement d'Organisation Nationale de la Guadeloupe], vous pouvez compter sur nous ".

15 La situation générale se dégrade rapidement entre affrontements, blocages, pillages et interpellations. Un responsable syndical est blessé par balle ${ }^{9}$, un autre syndicaliste est abattu sur un barrage $e^{10}$, un jeune sportif se tue à moto après avoir percuté un autre barrage. Dans ce climat de tension, la ministre de l'Intérieur et de l'Outre-Mer, Michèle 
Alliot-Marie annonce l'envoi supplémentaire de forces de l'ordre sur place; des personnes publiques (le footballeur Lilian Thuram, le chanteur Admiral T, etc.) lancent un appel à la non-violence; le premier ministre, François Fillon évoque la prise en compte des revendications par le gouvernement; le président de la République, Nicolas Sarkozy annonce l'ouverture d'états-généraux en Guadeloupe dès le retour au calme ${ }^{11}$.

Sous l'œil des caméras, des milliers de personnes viennent se recueillir et soutenir la famille du syndicaliste abattu, dans la chapelle ardente dressée au quartier général du LKP. Plusieurs personnalités de la gauche française apportent leur soutien au Collectif, certaines viennent sur place comme le leader du Nouveau Parti Anticapitaliste, Olivier Besancenot, ou encore les candidats aux élections présidentielles de 2007 en France, la socialiste Ségolène Royal et l'altermondialiste, José Bové.

\section{Oppositions à l'ordre médiatique national et local}

17 La grève générale éclate en Martinique le 5 février 2009, sous l'impulsion du Collectif $d u$ 5 février, exclusivement composé d'organisations syndicales. Les rues de Fort-de-France connaissent d'impressionnantes manifestations qui favorisent l'ouverture de négociations entre les représentants de l'État sur place, les élus, le patronat et le Collectif.

\section{Canal +et son regard « national » sur une réalité locale}

18 La tension remonte rapidement cependant, avec la télédiffusion d'un documentaire sur les héritiers blancs des premiers colons installés sur l'île avant la Révolution française ${ }^{12}$. Dans ce documentaire de 52 minutes $^{13}$, Alain Huygues-Despointes, entrepreneur béké regrette que les historiens ne s'intéressent pas "aux bons côtés de l'esclavage » et explique "vouloir préserver la race». Malgré les excuses qu'il présente et les regrets qu'il exprime a posteriori, les réactions hostiles aux békés ne font que croître depuis la diffusion antérieure du documentaire en France continentale et l'accès aux extraits de l'émission sur Internet. Le secrétaire d'État à l'Outre-mer dénonce des propos racistes, tandis que le parquet de Martinique ouvre une information judiciaire pour « apologie de crime contre l'humanité et incitation à la haine raciale». Le carnaval est annulé, une situation inédite depuis l'éruption de la montagne Pelée en 1902.

\section{Les dynamiques médiatiques du territoire}

Un autre élément significatif de la médiatisation de la grève générale en Martinique est la création de la Télévision Otonom Mawon (ТОМ). Ce média «engagé » dans le mouvement social de 2009 illustre une opposition à l'ordre médiatique établi localement et nationalement. Cette forme d'expression locale ne dure que le temps $\mathrm{du}$ conflit, elle prend le pas sur les émissions formatées depuis l'extérieur. Les programmes de la TOM sont diffusés par le web et en hertzien (Pulvar, 2013b).

Par ailleurs, à l'initiative d'un groupe d'intellectuels antillais, la rédaction du Manifeste sur les produits de haute nécessité souligne que cette grève générale contre les profits abusifs réalisés par le patronat local présente une dimension socioéconomique liée à une vieille revendication d'égalité de justice sociale. Le document publié dans la presse locale, nationale et internationale, largement diffusé en plusieurs langues et par Internet notamment, donne une dimension sociopolitique claire du conflit en rapport à 
la fois avec une critique du système capitaliste colonial et un projet de société Olivier Pulvar2015-07-20T18:18:000POlivier Pulvar2015-04-17T12:30:000PFarrah Berube2014-09-24T13:34:00(Breleur et al, 2009).

21 Ce que les médias nationaux ont présenté comme la "grève générale aux Antilles ", a éclaté dès le 5 décembre 2008 en Guadeloupe, suivi du temps fort du 20 janvier 2009. La mobilisation touche la Martinique, le 5 février avant de secouer d'autres territoires français d'outre-mer. La Guyane s'était déjà mobilisée en fin 2008 contre le prix des carburants et s'est fait entendre à travers un Front pour l'Avenir de la Guyane. La Réunion en proie aux tensions à partir du 13 février, a fait irruption dans ce conflit par l'intermédiaire du Collectif des organisations syndicales et politiques de la Réunion (Cospar).

Tous ces mouvements évoluent dans des contextes locaux proches, dans un contexte national partagé (plusieurs manifestations parisiennes bénéficiant du soutien de personnalités politiques nationales), et présentent des points communs dans leurs manifestations. Si cette crise sociale d'une ampleur sans précédent pour tous ces territoires, prend le nom de "grève générale aux Antilles ", c'est que la Guadeloupe et la Martinique sont les plus touchées par des mobilisations longues, dures et larges dans leurs manifestations comme dans leurs répercussions.

\section{Sur l'appropriation et les usages des médias par des groupes minoritaires}

Le mouvement \#YoSoy132 et la grève générale aux Antilles représentent des cas intéressants d'appropriation de l'espace et des contenus médiatiques par des groupes minoritaires dans des contextes de perturbation sociale. Le courant latino-américain des cultural studies offre un cadre théorique permettant d'étudier l'appropriation et les usages des médias par les acteurs.

Le courant latino-américain des cultural studies envisage la communication de masse comme un espace décisif dans lequel la sphère publique se redéfinit et la démocratie se reconstruit. L'analyse ne porte donc pas sur la communication de masse en tant que marchés et produits de consommation; elle fait plutôt ressortir les différentes manières dont les Latino-américains s'approprient les médias ainsi que les différents usages qu'ils en font. La communication et les industries culturelles sont saisies telle une matrice dans les doubles cas de perturbation et de réorganisation de l'expérience sociale (Martín-Barbero, 2012, pp. 209-210).

\section{Du médiactivisme de \#YoSoy132}

Dans le but de faire entendre sa voix, le mouvement mexicain \#YoSoy132 s'est approprié divers médias relevant des TIC. Les télévisions traditionnelles au Mexique étaient imperméables aux opinions s'opposant au PRI et les groupes minoritaires devaient ainsi investir l'espace médiatique via de nouveaux médias. \#YoSoy132 s'est doté d'un site internet, de micros blogs, de pages internet de réseaux sociaux, de sites de partage, de la messagerie et de la baladodiffusion. C'est la branche media du mouvement qui est en charge des opérations médiatiques ainsi que d'offrir aux adhérents du mouvement de nouvelles plateformes médiatiques pour s'exprimer. 
Dans son appropriation des médias, le mouvement anime donc un site internet : http:// www.yosoy132media.org. On trouve notamment sur ce site des actualités sur le mouvement, son historique, ses principes, sa charte, des vidéos, des archives, des pétitions à signer, des liens vers les autres médias du mouvement et des annonces diverses quant aux prochaines assemblées et manifestations. Un compte Twitter officiel appartient au mouvement et il compte 42000 abonnés $^{14}$. Des dizaines d'autres discussions twitter sont également recensées, celles-ci n'étaient plus actives en 2014 et elles n'étaient pas des discussions officielles du mouvement. Quelques pages Facebook (5 officielles) dont une destinée aux Mexicains habitants New York sont dénombrées. La plus officielle des pages Facebook du mouvement, https://www.facebook.com/yosoy132, compte 131,232 mentions «J'aime ${ }^{15}$. La seconde page officielle, https:// www.facebook.com/yosoy132media compte 28,990 mentions "J'aime» ${ }^{16}$. Le mouvement possède une chaîne YouTube officielle qui possède peu de vidéos mais dont la principale, celle du manifeste, compte 375,434 vues $^{17}$. Le mouvement dispose également des sites de partage wiki pour ses cellules à l'international, http:// yosoy132internacional.wikispaces.com, et un autre dédié à la définition du mouvement, http://en.wikipedia.org/wiki/Yo_Soy_132. Parmi les divers moyens de messagerie, une adresse de courriel et un compte Skype sont mis en place. Enfin le mouvement émarge aux médias traditionnels avec une émission sur Radiozapote qui peut être écoutée en baladodiffusion à partir du site internet officiel du mouvement.

L'appropriation d'une partie de l'espace médiatique par \#YoSoy132 a par conséquent réussi à perturber la campagne présidentielle de 2012. Les voix opposées aux vieux partis ont toujours existé au Mexique, comme partout ailleurs, elles ont trouvé dans les nouvelles plateformes médiatiques des espaces pour s'exprimer. Avant cette campagne, ces voix étaient peu relayées dans les médias traditionnels du Mexique alors que des liens antidémocratiques existaient entre partis politiques et médias. Le mouvement, d'abord étudiant, est venu déranger la quiétude de la campagne et ce grâce aux plateformes des nouveaux médias ayant permis le partage rapide et l'adhésion des masses (Bérubé, 2015).

Les tendances sur les intentions de vote en début de campagne et les résultats aux élections montrent que la campagne a en effet été perturbée. Nieto, le candidat du PRI à la présidence de la République, dominait tous les pronostics. En mai 2012, le quotidien El Universal publiait un sondage réalisé par la firme Buendía \& Laredo dans lequel Nieto était en tête avec $49,6 \%$ des intentions de vote ${ }^{18}$. Lors du vote le $1^{\text {er }}$ juillet 2012 , il ne remporte que le tiers des voix, soit $38,21 \%$. Pourtant troisième dans les intentions de vote, c'est le parti représentant une nouvelle coalition de trois partis de gauche et dirigé par Andres Manuel Lopez Obrador, ancien maire de México, qui arrive deuxième le jour des élections avec $31,59 \%$ des suffrages. Ce parti détenait pourtant seulement $24,8 \%$ des intentions de vote en mai 2012 selon le même sondage commandé par le quotidien El Universal. Le PAN, avec Josefina Vazquez Mota, arrivé troisième le jour des élections avec $25,41 \%$ des voix, perd le pouvoir qu'il détenait depuis 2000. Mota détenait $23,1 \%$ des intentions de vote en mai 2012 selon le sondage commandé par le quotidien El Universal. Cette dernière n'a donc pas bougé dans les intentions de vote et les résultats du vote. Il apparaît que les voix perdues du côté de Nieto se sont reportées sur Obrador. \#YoSoy132, sans favoriser aucun parti, a tout de même exprimé une opposition envers le gouvernement de Nieto et une adhésion à l'équipe de Obrador. 


\section{Aux scénographies antillaises de la citoyenneté}

29 Ce sont des mouvements sociaux d'ampleur qui secouent les Outremers français et notamment les Antilles au $1^{\text {er }}$ trimestre de l'année 2009. Ils posent la question de nouveaux rapports sociaux à l'intérieur de chacune des sociétés locales, de même que dans leurs relations entre elles; ces mouvements interrogent aussi la relation problématique qu'entretiennent ces territoires avec la France.

Nombre de pratiques collectives observées durant cette grève générale renforcent l'idée d'un profond malaise dans les Outremers français. Parmi celles-ci, les formes $d$ 'appropriation des médias et les usages des TIC apparaissent comme des ressources stratégiques pour la construction des identités culturelles ainsi que la transformation des rapports sociaux.

31 Au plan des appropriations des espaces et des contenus médiatiques, les publics, porteurs des identités minoritaires (antillais) développent un fort sentiment de frustration en réaction à la médiatisation nationale de la mobilisation sociale aux Antilles. Ils ressentent d'abord, une différence de traitement de leurs affaires d'avec celles des autres composantes de la République (sous-exposition), ensuite ils expriment leur insatisfaction du traitement de l'information (surexposition). Ils développent le même sentiment en réaction aux médiatisations locales entre scénographie de confrontation culturelles (identités majoritaire/minoritaires) et rétablissement de l'ordre médiatique (censure). Ils sont rejoints par des professionnels opposés aux schémas médiatiques dominants, qui développent en résonance avec les attentes de ces publics, une expression médiatique du mouvement social usant tant des médias classiques que des TIC (Télé Otonom Mawon, Kanal Martinique Télévision, Canal 10) (Pulvar, 2013a, 2013b).

32 Au plan des usages des TIC, les utilisateurs de réseaux sociaux numériques et de téléphones portables encouragent la circulation de récits en tout genre, de photos et vidéos sur et autour de la mobilisation. Ils créent un intense maillage de contenus qui, par leur variété, leur instantanéité et leur pertinence, complète voire supplée l'offre professionnelle. Lorsqu'on interroge la place des blogs, forums, ou autres plateformes virtuelles, ou autres dans le paysage médiatique, on note une forte volonté pour une parole ordinaire de se démarquer des circuits traditionnels de l'information et de s'afficher en tant que parole engagée et libre (Almar, 2012). Plusieurs types d'usage des blogs se dégagent : ceux animés par des journalistes professionnels et qui observent les règles de la profession tout en proposant une offre complémentaire aux médias traditionnels; ceux animés par des non professionnels de l'information qui dénoncent la censure et proposent en permanence des voies d'accès à l'information non (encore) diffusée officiellement; ceux qui rappellent aux professionnels de l'information les règles déontologiques et développent un rôle critique du journalisme ${ }^{19}$.

Les organisations syndicales mobilisées n'hésitent pas à solliciter les ressources des médias et des TIC par l'intermédiaire des Collectifs pour faire adhérer au-delà de leurs militants et sympathisants (le collectif du 5 février, http:// collectif5fevrier.blogspot.com/ ; le collectif Continuité LKP, https://sites.google.com/ site/continuitelkp/ ; le LKP http://ugtg.org/).

La mobilisation collective contre l'exploitation outrancière aux Antilles françaises représente un cas singulier d'appropriation de l'espace médiatique par des groupes minoritaires dans des contextes sociaux locaux fortement troublés. Suivant le principe 
de réseaux communautaires traditionnels, les appropriations des médias et les usages des TIC présentent une forme et un contenu très homogènes de mobilisations fondées sur des acteurs et des revendications très hétérogènes. La dynamique du Liyannaj structure les divers groupes et leur permet d'organiser la contestation.

Les formes d'appropriation et d'usage observées en vue de mobilisations citoyennes mettent en jeu divers facteurs : une conscience de la différence culturelle à laquelle on est très fortement attaché (avec parfois pour les plus jeunes, la fierté d'être noir), une solidarité dormante qui s'exprime de manière conjoncturelle, une volonté de participation rapidement mobilisable grâce à l'existence de réseaux organisés autour d'un sentiment d'appartenance, un rejet global de la violence. À travers leur forme de mobilisation politique, par les pratiques qu'ils développent autour des médias et des TIC, les acteurs créent des significations, des usages et des appropriations dans les relations qu'ils entretiennent à l'intérieur comme à l'extérieur de leurs territoires.

Les mobilisations collectives antillaises portent une exigence de respect, de solidarité et de justice sociale. Plusieurs demandes s'en dégagent : la reconnaissance de l'histoire coloniale de la France, la visibilité culturelle et identitaire des minorités nationales, l'ouverture du débat public sur les exclusions et les discriminations. Quelles qu'en soient les formes et les lieux d'expression publique, la critique du modèle français d'intégration s'inscrit dans une volonté de transformer le système.

Dans la perspective de Martin-Barbero, on peut interroger la dimension disproportionnée et paradoxale du rôle des médias aux Antilles, et l'observer plus généralement dans les Outremers français. En effet, les travaux de Watin (2001) appliqués aux espaces francophones et créolophones:

« (...) soulignent la pertinence d'envisager la médiatisation comme un phénomène qui favorise les conditions du débat sur l'identité collective et l'apparition d'une opinion publique. Elles privilégient une approche des médias à travers leur fonction identitaire dans l'espace public, en observant que leurs productions permettent à la fois la promotion de l'identité et l'émergence d'un espace public local. Les publics ont la possibilité de choisir leurs consommations (constitution identitaire des publics) et leur interprétations (émergence de communautés de réception) en dehors des canaux institutionnels (...)» (Pulvar, 2009c, p. 219).

Il serait intéressant d'élargir ce questionnement à d'autres espaces francophones qui connaissent des phénomènes de minoration culturelle.

De même, cette interrogation sur la dimension disproportionnée et paradoxale du rôle des médias aux Antilles pourrait sans doute s'appliquer à leur aire géographique et culturelle immédiate. Sur cette base, on tenterait de repérer une réflexion caribéenne en ce sens, au sein même du courant latino-américain des cultural studies. L'intérêt porterait sur les territoires insulaires de la Caraïbe (situation géographique) : produits de la colonisation et de l'esclavage (fait historique), essentiellement anglophones, hispanophones ou francophones avec la présence de langues vernaculaires (système sociolinguistique), Etats indépendants ou territoires dépendants de pays européens et étatsuniens (administration politique), exprimant des besoins, présentant des inégalités et des vulnérabilités, comptant des dispositifs de communication (demandes/ organisation sociale). 


\section{Vers de nouvelles manières de faire ensemble?}

La mobilisation du mouvement \#YoSoy132 comme celles des Collectifs LKP et du 5 février montrent que les crises contemporaines affectent la circulation de l'information, notamment dans leurs répercussions sociales. Les modes de diffusion classiques des médias de masse se mêlent à ceux plus émergents des réseaux électroniques. Autour des sources officielles ou autorisées gravitent une pluralité d'émetteurs, une variété de contenus, une diversité des voies et moyens de communication qui favorisent les appropriations et usages stratégiques des acteurs.

Dans un contexte mondialisé, l'action des technologies transforme les cultures: une continuité du sens (idées, croyances, opinions,) prenant forme dans des espaces symboliques se substitue à la discontinuité des territoires et du temps évoluant dans des espaces physiques. Les processus industriels donnent une nouvelle résonance aux mobilisations socioculturelles et politiques et ils redéfinissent les identités en relation. Par conséquent, les phénomènes de communication en œuvre dans des espaces sociaux troublés indiquent davantage la nature des mutations en cours qu'une action mécanique de modernisation politique ou technique.

Les travaux menés au Mexique et aux Antilles dans cette perspective permettent de revisiter la question du rôle des médias et des TIC, au-delà de l'approche par les effets. Dès lors, convenons que les médias agissent sur les cultures et les identités au moins autant que ces dernières en réinterprètent les messages pour les faire fonctionner dans l'espace social.

Aussi, la place des médias et des TIC dans les mobilisations révèle de nouvelles formes collectives de traitement de l'information, d'engagement et de participation des acteurs dans le réel social. Leurs appropriations et usages par des groupes minoritaires peut contrarier l'information officielle au profit d'une parole populaire libérée qui propose de faire autre chose, de faire autrement pour mieux être à l'intérieur de son territoire.

\section{BIBLIOGRAPHIE}

Bibliographie

Almar, Nathalie, «Quels usages des blogs dans la crise antillaise de 2009 ? Interroger le rôle des publics dans la production de l'information », Usages et pratiques des publics dans les pays du Sud. Des médias classiques aux TIC, Actes du colloque international pluridisciplinaire, Agadir, 2012, p. 327-332.

Antiope, Nathalie et Pulvar, Olivier, «Les identités régionales à l'épreuve de la TNT », CRPLCCNRS UMR 8053, Université Antilles-Guyane, papier de recherche, 2010, 13 p.

Arciniegas, Germán, Biografgas del Caribe, Bogota, Plaza \& Janes, 429 p. 
Bérubé, Farrah, « \#YoSoy132, la mobilisation citoyenne mexicaine qui a secoué les élections présidentielles de 2012 »In Médias et nouvelles formes d'engagement sociopolitique, Fathallah Daghmi (dir.), Paris, l'Harmattan, 2015, à paraître.

Breleur, Ernest et al, Manifeste pour les produits de haute nécessité, Martinique - Guadeloupe - Guyane Réunion, Paris, Editions Galaade /Institut du Tout-monde, 2009, 16 p.

Calimia-Dinane, Nathalie, « Le mouvement social en Guadeloupe, 20 janvier 2009-4 mars 2009 », La Guadeloupe en bouleverse, Pointe-à-Pitre, Ed. Jasor, 2009, p. 29-36.

Martin-Barbero, Jesús, « A Latin American Perspective on Communication/Cultural Mediation », In International Communication, Daya Kishan Thussu (ed.), vol. 4, Los Angeles, Sage, 2012, p. 205-220.

Pulvar, Olivier, « Communications et identités diasporiques des Antillais en France », In Agbobli Christian, Oumar Kane, Gaby Hsab (dir.), Identités diasporiques et communication, Québec, PUQ, 2013a, p. 71-86.

Pulvar, Olivier, « Montrer différemment pour se regarder autrement : la TOM, un « média éphémère » dans le mouvement social de 2099 en Martinique », Asylon(s), nº11, mai 2013, Quel colonialisme dans la France d'outre-mer ?, 2013b, [en ligne] http://www.reseau-terra.eu/ article1276.html, consulté le 7/4/2015.

Pulvar, Olivier, « Constructions identitaires et communications médiatisées en Martinique », In Idelson Bernard, Magdelaine-Andrianjafitrimo Valérie (dir.), Paroles d'outre-mer. Identités linguistiques, expressions littéraires, espaces médiatiques, Paris, l'Harmattan, 2009c, p. 215-222.

Trigo, Abril, « General Introduction » in The Latin American Cultural Studies Reader, Ana del Sarto, Alicia Ríos et Abril Trigo (éd.), Durham, Duke University Press, 2004, p. 1-14.

Watin, Michel, (dir.), Communication et espace public. Univers créoles 1, Paris, Anthropos/Economica, 2001, 266 p.

\section{NOTES}

1. Au début de la campagne, Enrique Peña Nieto récoltait $49,6 \%$ des intentions de vote. Source: http://www.infoeleccionesmexico.com/noticia-resultados-encuesta-eluniversal-mayo-147.html, consulté le 23/9/2013

\section{Assassin}

3. Dehors

4. http://www.youtube.com/watch?v=2ZI1JIURI9Q, consulté le 16/9/2013.

5. http://www.youtube.com/watch?v=P7XbocXsFkI, consulté le 16/9/2013.

6. http://www.youtube.com/watch?v=cfWj5da6Yhk, consulté le 16/9/2013.

7. http://www.animalpolitico.com/2012/05/declaratoria-y-pliego-petitorio-de-yosoy-132/\#axzz2f4kHmQw2, consulté le 16/9/2013.

8. Dans une communauté culturelle où les composantes linguistique créole et phénotypique noire sont majoritaires, on saisit pleinement l'effet désastreux des indices sonores et iconiques sur les publics lorsque c'est le groupe blanc (minoritaire à l'image comme dans l'espace public) qui se retire laissant en plan le groupe noir à la table. 
9. Les chaines locales retransmettent le témoignage du syndicaliste Alex Lollia qui déclare « Les CRS sont arrivés : ils m'ont dit sale chien! Sale nègre! On t'a vu hier soir à la télé, on va te casser !»

10. Jacques Bino est tué par balle sur un barrage tenu par des jeunes à Pointe-à-Pitre alors qu'il rentrait d'un meeting du LKP.

11. C'est la première fois depuis le début du conflit que Nicolas Sarkozy évoque la situation de crise en Outre-mer, et sur les seules ondes du Réseau France Outre-mer ; il promet de répondre à "une certaine forme de désespérance de nos compatriotes des territoires d'Outre-mer ».

12. Il faut rappeler que depuis 2008, un bouquet satellite payant (Canal+, Canal Sat Antilles), élargit le spectre de réception de l'information au plan régional, permettant aux téléspectateurs d'un même territoire de recevoir les chaînes publiques des autres territoires, mais aussi des stations privées qui y sont implantées (Antiope, Pulvar, 2010; Pulvar, 2013b).

13. Réalisé par Romain Bolzinger, monté par Franck Zahler, « Les derniers maîtres de la Martinique » a été produit par TAC PRESSE, diffusé le 30 janvier 2009 dans Spécial Investigation sur Canal+ et le 6 février sur Canal+ Antilles.

14. En date du 14/1/2014.

15. En date du 14/1/2014.

16. En date du 14/1/2014.

17. En date du 17/9/2013.

18. http://www.infoeleccionesmexico.com/noticia-resultados-encuesta-el-universalmayo-147.html, consulté le 23/9/2013.

19. C'est ainsi que les acteurs légitiment leur place dans le système médiatique dont ils participent de la redéfinition.

\section{RÉSUMÉS}

Les crises contemporaines affectent la circulation de l'information, notamment dans leurs répercussions sociales. Les modes de diffusion classiques des médias de masse en côtoient d'autres, plus émergents, au centre desquels les réseaux électroniques occupent une position centrale. Dès lors, la pluralité des émetteurs de messages, la variété des contenus, la diversité des voies et moyens de la communication autour des phénomènes en cours s'ajoutent aux "sources autorisées ». Cette communication plurielle encourage des appropriations de contenus identitaires et des usages informatifs, symboliques, voire stratégiques des médias. Facteur parmi d'autres des mutations sociales et institutionnelles, l'effet des médias et/ou des TIC sur les réalités politiques et sociales est désormais admis. Plutôt que d'y observer l'action intrinsèque des techniques dans le sens du progrès des collectivités humaines, il faut voir dans les processus de communication, un indicateur des mutations en cours. Les travaux menés au Mexique et aux Antilles dans cette perspective permettent de revisiter la question du rôle des médias et des TIC dans ces sociétés, au-delà de l'approche par les effets privilégiée jusque-là. Les médias agissent 
sur les cultures et les identités au moins autant que ces dernières en réinterprètent les messages pour les faire fonctionner dans un espace social donné. Aussi, s'interroger sur la place concrète des médias et des TIC dans les mobilisations collectives, soulève une autre question : comment repérer et caractériser les nouvelles formes collectives de traitement de l'information, d'implication, de participation et de mobilisation?

Contemporary crisis modify the flow of information, especially in their social impact. Classical ways of diffusion of mass media now go alongside with emerging media, in which electronic networks occupy a central position. Since now, a plurality of issuers of messages, a variety of contents, a diversity of ways and tools of communication add to the "authorized sources" around the phenomenons in place. That plural communication encourages the appropriations of identity contents and informatives, symbolics and in some cases strategics uses of media. The impact of media and/or of ICT on political and social realities is now on recognized as one of the factors of social and institutional mutations. Instead of observing the intrinsic action of techniques in sense of progress for human collectivities, we must see in the processes of communication an indicator of mutations taking place. Studies directed in Mexico and in West Indies according to that perspective allow to rethink the question of the role of media and ICT in those societies beyond the effects approach privileged until now. Media act upon cultures and identities at least when they reinterpret the messages to make them work in a specific social sphere. Also, interrogating the concrete place of media and ICT in the collective mobilizations, points out another question: how identify and characterize the new collective forms of treatment of information, of implication, of participation and of mobilization?

Las crisis contemporáneas afectan a la circulación de información, especialmente en sus repercusiones sociales. Los modos de difusión clásicos de los medios de comunicación se codean con medios en emergencia, entre cuyos los redes electrónicos ocupan una posición central. Desde entonces, la pluralidad de emisores de mensajes, la variedad de contenidos, la diversidad de vías y de medios de comunicación, alrededor a los fenómenos en curso se añaden a las «fuentes autorizadas». Esta comunicación plural fomenta las apropiaciones de contenidos sobre las identidades y de usos informativos, simbólicos, incluso estratégicos de los medios de comunicación. Factor entre otros de mutaciones sociales e institucionales, el efecto de los medios de comunicación y/o de las tecnologías de información y de comunicación (TIC) sobre las realidades políticas y sociales está en adelante admitido. En lugar de observar ahí la acción intrínseca de la técnicas en el sentido del progreso de las colectividades humanes, hay que ver en los procesos de comunicación, un indicador de las mutaciones en curso. Los estudios conducidos en México y en la Antillas con esa perspectiva permiten de revisar la cuestión de la función de los medios de comunicación y de las TIC en estas sociedades, más allá del enfoque de los efectos privilegiado hasta ahí. Los medios de comunicación actúan sobre las culturales y las identidades al menos cuando estas últimas interpretan de nuevo los mensajes para que funcionan en un espacio social determinado. Además, preguntarse sobre el sitio concreto de los medios de comunicación y de las TIC en las movilizaciones colectivas, plantea otra pregunta: ¿cómo ver y caracterizar a las nuevas formas colectivas de tratamiento de la información, de implicación, de participación y de movilización?

\section{INDEX}

Keywords : Media, ICT, uses, appropriations, identities, cultures, globalization

Palabras claves : Medios de comunicación, tecnologías de información y de comunicación (TIC), usos, apropiaciones, identidades, culturales, internacionalización

Mots-clés : Médias, TIC, usages, appropriations, identités, cultures, mondialisation 


\section{AUTEURS}

\section{FARRAH BÉRUBÉ}

GERACII-UQAM, Université du Québec à Trois-Rivières (Québec, Canada), Bureau 4032, pavillon Ringuet, 3351. Boulevard. des Forges, C.P. 500, Trois-Rivières (Qc) G9A 5H7,

farrah.berube@uqtr.ca

\section{OLIVIER PULVAR}

CRPLC-CNRS UMR 8053, Université des Antilles (Martinique, France), Faculté de droit et d'économie, 97275 Schœlcher Cedex, opulvar@martinique.univ-ag.fr 